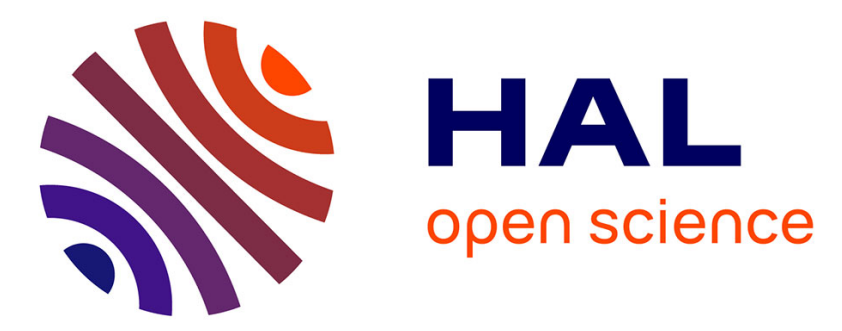

\title{
Nonlinear Control Design for Multi-Terminal Voltage-Sourced Converter High Voltage Direct Current Systems with Zero Dynamics Regulation
}

Yijing Chen, Weihuang Huang, Yan Li, Hong Rao, Shukai Xu, Gilney Damm

\section{- To cite this version:}

Yijing Chen, Weihuang Huang, Yan Li, Hong Rao, Shukai Xu, et al.. Nonlinear Control Design for Multi-Terminal Voltage-Sourced Converter High Voltage Direct Current Systems with Zero Dynamics Regulation. 4th IEEE Workshop on the Electronic Grid (eGRID 2019), Nov 2019, Xiamen, China. pp.9092694, 10.1109/eGRID48402.2019.9092694 . hal-02861769

\section{HAL Id: hal-02861769 https://hal.science/hal-02861769}

Submitted on 11 Sep 2020

HAL is a multi-disciplinary open access archive for the deposit and dissemination of scientific research documents, whether they are published or not. The documents may come from teaching and research institutions in France or abroad, or from public or private research centers.
L'archive ouverte pluridisciplinaire HAL, est destinée au dépôt et à la diffusion de documents scientifiques de niveau recherche, publiés ou non, émanant des établissements d'enseignement et de recherche français ou étrangers, des laboratoires publics ou privés. 


\section{Nonlinear Control Design for Multi-Terminal Voltage-Sourced Converter High Voltage Direct Current Systems with Zero Dynamics Regulation}

\author{
$1^{\text {st }}$ Yijing Chen, $3^{\text {rd }}$ Weihuang Huang, $4^{\text {th }}$ Yan Li, $5^{\text {th }}$ Hong Rao, $6^{\text {th }}$ Shukai Xu \\ State Key Laboratory of HVDC \\ Electric Power Research Institute, China Southern Power Grid \\ Guangzhou 51063, P.R. China \\ chenyj@csg.cn
}

\author{
$2^{\text {nd }}$ Gilney Damm \\ Laboratoire IBISC \\ Paris-Saclay University \\ Saclay, France \\ gilney.damm@centralesupelec.fr
}

\begin{abstract}
This paper presents a nonlinear control strategy with a nested control structure for a class of multi-terminal VSC HVDC systems. An output control law is first proposed, which is based on the passive output. When the output variables converge to zero with the developed output control law, the system behavior is governed by the uncontrolled zero dynamics. However, due to some desired control variables such as the DC voltage that belong to the internal dynamics, an outer control loop is then suggested to arbitrarily drive the zero dynamics, which provides a reference to be tracked by the output control law. The performance of the proposed control algorithm is evaluated by numerical simulations which show that the system has a much faster convergence rate than the controller without the outer loop.
\end{abstract}

Index Terms-Nonlinear control design, power converters, multi-terminal VSC HVDC systems

\section{INTRODUCTION}

In the past decades, significant advances have been achieved in the development of high power devices which can convert the current from AC to DC and vice versa [1]. This has provided beneficial conditions for the application of multi-terminal voltage-sourced converter (VSC) high voltage direct current (HVDC) systems which can realize power exchange between multiple power suppliers and multiple power consumers.

Control design is one of the most popular research topics in the field of HVDC systems. There are two well established control methods, namely direct and vector control methods, for controlling the VSC. The direct control method is very straightforward where the control variables, the phase angle and the amplitude modulation ratio, are directly derived from the measurements of the currents and the voltages at the point of common coupling (PCC) [2], [3]. The main drawback of this control approach is its incapability of limiting the current through the converter to protect the semiconductor devices. To overcome this shortcoming, the vector control method is proposed [4], [5], which is composed of a fast inner current loop and a slow outer loop. These two control methods are mainly based on several standard PI controllers and hence, extra efforts are usually needed to tune the PI control gains [6]. To develop more efficient control schemes, some nonlinear control techniques are used. In [7], a nonlinear model predictive control method is applied to the inner current loop without the need of a phase-locked loop (PLL). As presented in [8], [9], input-output feedback linearization is proposed for the control of VSC. However, the asymptotic stability of the system is determined by the asymptotic stability of the corresponding zero dynamics. There have been also several studies on the passivity-based control (PBC) design for a class of switched power converters which can be described in port-Hamiltonian form [10]-[13].

In the present paper, we propose a nonlinear control scheme for a class of Multi-Terminal VSC HVDC (MTDC) systems. Apart from the prior research work, we first introduce a passive output derived from a storage function and then, design a new output based on the passive output. Subsequently, we further investigate the corresponding zero dynamics of the designed new output. Several variables to be controlled belong to the zero dynamics, which convergence rate of the zero dynamics are determined by the system inherent characteristics. Most often, such natural convergence rates are far from acceptable, and for this reason, additional control inputs are introduced to modify these internal dynamics and improve their performances.

The reminder of this paper is outlined as follows. In Section II, the model of the proposed MTDC system is established in a synchronously rotating $d q$ reference frame. The proposed control scheme is developed in Section III where the zero dynamics are also discussed. The performance of the proposed control algorithm is evaluated in Section IV. Finally, the conclusions are drawn in Section V.

Notations: The element of matrix $A \in \mathbb{R}^{n \times m}$ in the $i^{\text {th }}$ row and the $j^{\text {th }}$ column is denoted by $A_{i j}$. The $i^{\text {th }}$ row and the $j^{\text {th }}$ column of $A$ are represented by $A(i,:)$ and $A(:, j)$, respectively. The transpose of $\mathrm{A}$ is denoted by $A^{T}$. The rank of $A$ is $\operatorname{rank}(A)$. A diagonal matrix $A$ can be represented by $A=\operatorname{diag}\left(a_{i}\right)$ whose diagonal elements are $a_{i}, i=1, \cdots, n$. $0_{n \times m} \in \mathbb{R}^{n \times m}$ represents the zero matrix, with all its elements equal zero. Unless otherwise stated, in this paper, $i, \rho j, k$ and $h$ mean " $\forall i \in \mathcal{N}$ ", " $\forall \rho \in \mathcal{N}_{-1}$ ", " $\forall j \in \mathcal{M}$ ", " $\forall$ $k \in \mathcal{L}$ " and " $\forall h \in \mathcal{P}$ ", respectively, where $\mathcal{N}=\{1, \cdots, N\}$, $\mathcal{N}_{-1}=\{2, \cdots, N\}, \mathcal{M}=\{1, \cdots, M\}, \mathcal{L}=\{1, \cdots, L\}$, 
$\mathcal{P}=\{1, \cdots, P\}$

\section{Modeling OF THE MTDC System}

A general configuration of an MTDC system with $N$ strong AC systems (SAC), $M$ weak AC systems (WAC) and a DC network is depicted in Fig. 1 where each $\mathrm{AC}$ network is connected by only one VSC converter.

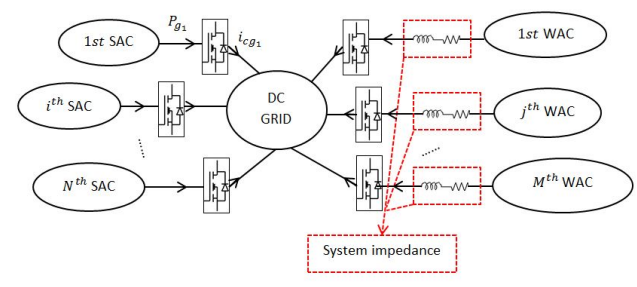

Fig. 1. A multi-terminal VSC HVDC system with strong and weak AC systems.

\section{A. Modeling of the SAC side}

The configuration of the $i^{\text {th }}$ SAC connection terminal is presented in Fig. 2 where the currents $i_{g_{i}, d q}$ flow through the phase reactor made up of an aggregated resistance $R_{g_{i}}$ and an aggregated inductance $L_{g_{i}}$. Since the SAC enables to control its AC voltage at the PCC in case of disturbances, the SAC can be modeled by an ideal three-phase $\mathrm{AC}$ source with constant parameters. Consequently, the AC voltage of the $i^{\text {th }} \mathrm{SAC}$, i.e. $v_{s g_{i}, d q}$, can be always maintained at fixed frequency $f_{g_{i}}$ and amplitude $V_{g_{i}, \text { rms }}$.

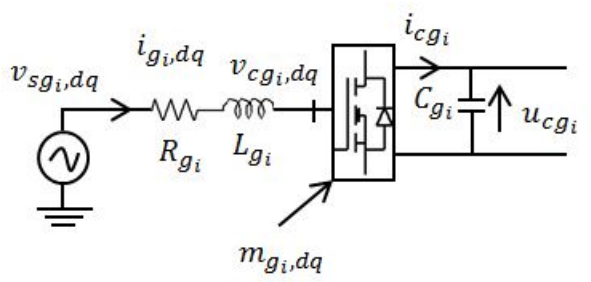

Fig. 2. A simplified schematic diagram of the $i^{\text {th }}$ VSC connected strong grid.

The dynamics of $i_{g_{i}, d q}$ are described by

$$
\begin{aligned}
\frac{d i_{g_{i} d}}{d t} & =-\frac{R_{g_{i}}}{L_{g_{i}}} i_{g_{i} d}+\omega_{g_{i}} i_{g_{i} q}+\frac{v_{s g_{i} d}}{L_{g_{i}}}-\frac{u_{c g_{i}}}{2 L_{g_{i}}} m_{g_{i} d} \\
\frac{d i_{g_{i} q}}{d t} & =-\frac{R_{g_{i}}}{L_{g_{i}}} i_{g_{i} q}-\omega_{g_{i}} i_{g_{i} d}+\frac{v_{s g_{i} q}}{L_{g_{i}}}-\frac{u_{c g_{i}}}{2 L_{g_{i}}} m_{g_{i} q}
\end{aligned}
$$

where $w_{g_{i}}=2 \pi f_{g_{i}}$ and the modulation indices $m_{g_{I}, d q}$ are the control variables.

In addition, according to the power balance on both sides of the converter station, the DC current $i_{c g_{i}}$ satisfies

$$
i_{c g_{i}}=\frac{3}{4}\left(m_{g_{i} d} i_{g_{i} d}+m_{g_{i} q} i_{g_{i} q}\right)-\frac{u_{c g_{i}}}{R_{\text {equi }, g_{i}}}
$$

where $R_{\text {equi,(.) }}$ represent the losses of the converters. For the sake of simplicity, the $d q$ reference frame is usually chosen such that the $d$-axis is aligned to the phase $a$ of AC voltage, which results in $v_{s g_{i} d}=V_{g_{i}, \text { rms }}$ and $v_{s g_{i} q}=0$. As a result, the instantaneous active power $P_{g_{i}}$ and reactive power $Q_{g_{i}}$ at the PCC are given by:

$$
P_{g_{i}}=\frac{3}{2} v_{s g_{i} d} i_{g_{i} d}, Q_{g_{i}}=-\frac{3}{2} v_{s g_{i} d} i_{g_{i} q}
$$

$R_{\text {equi,(.) }}$ represent the losses of the converters.

\section{B. Modeling of the WAC side}

A simplified schematic diagram of the $j^{\text {th }}$ WAC connection terminal is shown in Fig. 3. Such weak grids can be modelled as renewable energy sources like wind farms. In this case, an aggregated output is used to represent all individual wind turbine generator's outputs. In addition, we consider that every wind turbine is based on doubly-fed induction generator $(\text { DFIG })^{1}$ [14]. Therefore, the wind farm can be modeled as a controlled source described by $I_{w_{j}, d q}$ [15], [16]. Due to the use of step-up transformers, some high-order harmonics are produced. Consequently, the high frequency AC filter represented by a simple capacitor $C_{f w_{j}}$ is used to eliminate those unacceptable harmonics.

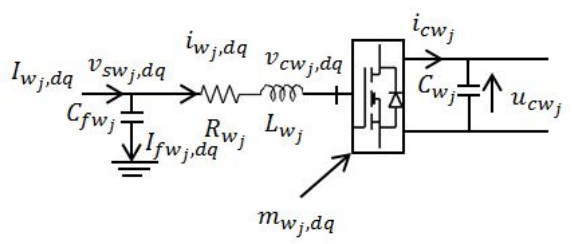

Fig. 3. A simplified schematic diagram of the $j^{\text {th }}$ VSC connected weak grid.

The basic equation for the $d q$ current $i_{w_{j}, d q}$ at the PCC is given by

$$
\begin{aligned}
& \frac{d i_{w_{j} d}}{d t}=-\frac{R_{w_{j}}}{L_{w_{j}}} i_{w_{j} d}+\omega_{w_{j}} i_{w_{j} q}+\frac{v_{s w_{j} d}}{L_{w_{j}}}-\frac{u_{c w_{j}}}{2 L_{w_{j}}} m_{w_{j} d} \\
& \frac{d i_{w_{j} q}}{d t}=-\frac{R_{w_{j}}}{L_{w_{j}}} i_{w_{j} q}-\omega_{w_{j}} i_{w_{j} d}+\frac{v_{s w_{j} q}}{L_{w_{j}}}-\frac{u_{c w_{j}}}{2 L_{w_{j}}} m_{w_{j} q}
\end{aligned}
$$

and the dynamics of the $d q$ voltage $v_{w_{j}, d q}$ at the PCC are modeled as

$$
\begin{aligned}
& \frac{d v_{s w_{j} d}}{d t}=\omega_{w_{j}} v_{s w_{j} q}+\frac{1}{C_{f w_{j}}}\left(I_{w_{j} d}-i_{w_{j} d}-\frac{v_{s w_{j} d}}{R_{f w_{j}}}\right) \\
& \frac{d v_{s w_{j} q}}{d t}=-\omega_{w_{j}} v_{s w_{j} d}+\frac{1}{C_{f w_{j}}}\left(I_{w_{j} q}-i_{w_{j} q}-\frac{v_{s w_{j} q}}{R_{f w_{j}}}\right)
\end{aligned}
$$

where the equivalent resistance $R_{f w_{j}}$ is used to represent the losses of the filter.

In addition, the active and the reactive power of the WAC terminal at the PCC are expressed as

$$
\begin{aligned}
& P_{w_{j}}=\frac{3}{2}\left(v_{s w_{j} d} i_{w_{j} d}+v_{s w_{j} q} i_{w_{j} q}\right) \\
& Q_{w_{j}}=\frac{3}{2}\left(v_{s w_{j} q} i_{w_{j} d}-v_{s w_{j} d} i_{w_{j} q}\right)
\end{aligned}
$$

Similar to $i_{c g_{i}}$, the DC current of the WAC terminal $i_{c w_{j}}$ satisfies

$$
i_{c w_{j}}=\frac{3}{4}\left(m_{w_{j} d} i_{w_{j} d}+m_{w_{j} q} i_{w_{j} q}\right)-\frac{u_{c w_{j}}}{R_{\text {equi }, w_{j}}}
$$

\footnotetext{
${ }^{1}$ One of the most common technology used for large turbines.
} 


\section{Modeling of the DC network}

A generic DC network topology is formed by $N$ SAC converter nodes, $M$ WAC converter nodes, $P$ intermediate nodes and $L$ transmission branches [17]. As depicted in Fig. 4, the $i^{\text {th }}$ SAC converter node, the $j^{\text {th }}$ WAC converter node and the $h^{\text {th }}$ intermediate node are characterized by their corresponding DC voltages, i.e. $u_{c g_{i}}, u_{c w_{j}}$ and $u_{c t_{h}}$, and DC capacitors, $C_{g_{i}}$ and $C_{w_{j}}$ and $C_{t_{h}}$. The $k^{\text {th }}$ branch transmission line $l_{k}$ is modeled by a lumped $\pi$-equivalent circuit [18] composed of the aggregated resistance $R_{c_{k}}$ and inductance $L_{c_{k}}$. The branch current of $l_{k}$ is denoted as $i_{c_{k}}$. Every branch circuit is used to connect two adjacent nodes and every node can be connected to a number of transmission lines. As illustrated in Fig. 4, the green arrow means that the branch circuit current is fed into the node, whereas the violet one represents the branch circuit current discharges from the node.

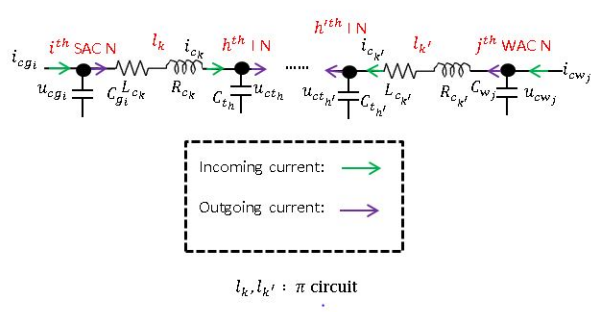

Fig. 4. DC circuit.

In order to better understand the properties of the DC network, we analyze its topology structure with the help of graph theory. In this paper, we study a class of DC networks which can be represented by a weakly connected directed graph $G$ without self-loops. This graph can be labeled by $G=(V, E) . V=\left\{V_{1}, V_{2}, V_{3}\right\}$ is the set of the vertices where $V_{1}=\left\{v_{1}, \cdots, v_{N}\right\}, V_{2}=\left\{v_{N+1}, \cdots, v_{N+M}\right\}$ and $V_{3}=\left\{v_{N+M+1}, \cdots, v_{N+M+P}\right\}$ correspond to the $N$ SAC converter nodes, the $M$ WAC converter nodes and the $P$ intermediate nodes, respectively. $E=\left\{e_{1}, \cdots, e_{L}\right\}$ is the set of the edges mapping to the $L$ circuit branches. The incidence matrix of $G=(V, E)$ is denoted by $H \in \mathbb{R}^{(N+M+P) \times L}$ and its element in the $l^{\text {th }}$ row and the $k^{\text {th }}$ column, i.e. $H_{l k}$, satisfies

$$
H_{l k}= \begin{cases}1 \quad & \text { if the branch current of } e_{k} \text { flows } \\ & \text { into the node } v_{l} \\ -1 \quad & \text { if the branch current of } e_{k} \text { flows } \\ \text { from the node } v_{l} & \\ 0 \quad \text { otherswise }\end{cases}
$$

Hence, based on (2) and (7), the dynamics of the DC grid can be written in matrix expression form as

$$
\dot{z}=A_{p} z+\vartheta_{p}
$$

with the following notations

$$
\begin{aligned}
z= & {\left[\begin{array}{ll}
u_{c} & i_{c}
\end{array}\right]^{T} \in \mathbb{R}^{N+M+P+L} } \\
u_{c}= & {\left[\begin{array}{lll}
u_{c g} & u_{c w} & u_{c t}
\end{array}\right]^{T} } \\
u_{c g}= & {\left[\begin{array}{lll}
u_{c g_{1}} & \cdots & u_{c g_{N}}
\end{array}\right]^{T} } \\
u_{c w}= & {\left[\begin{array}{lll}
u_{c w_{1}} & \cdots & u_{c w_{M}}
\end{array}\right]^{T} } \\
u_{c t}= & {\left[\begin{array}{lll}
u_{c t_{1}} & \cdots & u_{c t_{P}}
\end{array}\right]^{T} } \\
i_{c}= & {\left[\begin{array}{lll}
i_{c_{1}} & \cdots & i_{c_{L}}
\end{array}\right]^{T} } \\
\vartheta_{p}= & {\left[\begin{array}{lll}
\cdots & \frac{3\left(m_{g_{i} d} i_{g_{i} d}+m_{g_{i} q} i_{g_{i} q}\right)}{4 C_{g_{i}}} \cdots \\
& \cdots \\
& \frac{3\left(m_{w_{j} d} i_{w_{j} d}+m_{w_{j} q} i_{w_{j} q}\right)}{4 C_{w_{j}}} \cdots 0_{(P+L)}
\end{array}\right]^{T} }
\end{aligned}
$$

The matrix $A_{p}$ is of the form

$$
A_{p}=\left[\begin{array}{cc}
-D_{p} & C^{-1} H \\
-L^{-1} H^{T} & -L^{-1} R
\end{array}\right]
$$

where $C \in \mathbb{R}^{(N+M+P) \times(N+M+P)}$ and $L, R \in \mathbb{R}^{L \times L}$ are the capacitor, inductance and resistance matrices, respectively, which are given by

$$
\begin{aligned}
C & =\operatorname{diag}\left(C_{g_{1}} \cdots C_{g_{N}} C_{w_{1}} \cdots C_{w_{M}} C_{t_{1}} \cdots C_{t_{P}}\right) \\
L & =\operatorname{diag}\left(L_{c_{1}} \cdots L_{c_{L}}\right) \\
R & =\operatorname{diag}\left(R_{c_{1}} \cdots R_{c_{L}}\right) \\
D_{p} & =C^{-1} R_{\text {equi }}^{-1} \\
R_{\text {equi }} & =\operatorname{diag}(\cdots, R_{\text {equi } g_{i}}, \cdots R_{\text {equi }, w_{j}}, \cdots, \underbrace{0, \cdots, 0}_{P+L})
\end{aligned}
$$

Remark 1: It is worthwhile to keep in mind that taking into account physical considerations [19], the feasible region of the state variables is not boundless. Therefore, in this paper, we restrict the state variables to some domains of interest. All DC voltage $u_{c g_{i}}, u_{c w_{j}}$ and $u_{c t_{h}}$ are considered on the domain $\mathbb{D}_{u_{c}} \triangleq\left[u_{c, \text { min }}, u_{c, \text { max }}\right] \in \mathbb{R}$ where $u_{c, \text { min }}$ is positive. The current $i_{g_{i}, d q}$ and $i_{w_{j}, d q}$ are defined on $\mathbb{D}_{i_{i}, d q} \in \mathbb{R}$ and $\mathbb{D}_{i_{w_{j}, d q}} \in \mathbb{R}$, respectively. All branch currents $i_{c_{k}}$ are on the domain $\mathbb{D}_{i_{c}}$. Consequently, the domains $\mathbb{D}_{(\cdot)}$ are defined as the domains of interest, which are bounded.

\section{CONTROL SCHEME FOR THE MTDC SYSTEM}

In order to keep the DC voltage within the acceptable band, at least one of the converter stations in the MTDC system must be used to regulate the DC voltage. In this paper, master-slave control configuration is used where a single converter terminal is assigned to control the DC voltage at a constant level and the rest of the converter stations work in other control modes. Since the master terminal takes responsibility for balancing the power flow of the DC network in case of disturbances, this terminal usually needs a large power capacity to counteract all possible power imbalance.

The arrangement of VSC operation for the MTDC system is set as follows:

- The $1^{\text {st }}$ SAC converter station chosen as the master terminal is used to maintain the DC voltage at the constant level $u_{c g_{1}}^{o}$ while the remaining SAC converter stations 
operate in active control mode to control $P_{g_{\rho}}$ at $P_{g_{\rho}}^{o}{ }^{2}$. Additionally, all reactive power $Q_{g_{i}}$ should be kept at their respective references $Q_{g_{i}}^{o}$.

- Each WAC converter station must ensure that the magnitude and the frequency of the AC voltage at the PCC, $V_{s w_{j}, \text { rms }}$ and $f_{w_{j}}$, are kept constant. This can be fulfilled by regulating $v_{s w_{j}, d q}$ at their constant references $v_{s w_{j}, d q}^{o}$.

In order to make all SAC systems operate with unity power factor, we set

$$
Q_{g_{i}}^{o}=0
$$

\section{A. Steady state analysis}

Consider the system $\Sigma_{1}$ described by (1), (4), (5) and (9) with the output variables $u_{c g_{1}}, i_{g_{\rho} d}, i_{g_{i} q}$ and $v_{s w_{j}, d q}$. For the prescribed references $u_{c g_{1}}^{o}, Q_{g_{1}}^{o}, P_{g_{\rho}}^{o}, Q_{g_{\rho}}^{o}, v_{s w_{j}, d q}^{o}$, the equilibrium point of $\Sigma_{1}$ denoted by $\bar{P}_{1}$ exists and the steadystate values denoted by $(\bar{\cdot})$ of the state variables satisfy

$$
\begin{aligned}
& 0=-\frac{R_{g_{i}}}{L_{g_{i}}} \bar{i}_{g_{i} d}+\omega_{g_{i}} \bar{i}_{g_{i} q}+\frac{v_{s g_{i} d}}{L_{g_{i}}}-\frac{\bar{u}_{c g_{i}}}{2 L_{g_{i}}} \bar{m}_{g_{i} d} \\
& 0=-\frac{R_{g_{i}}}{L_{g_{i}}} \bar{i}_{g_{i} q}-\omega_{g_{i}} \bar{i}_{g_{i} d}+\frac{v_{s g_{i} q}}{L_{g_{i}}}-\frac{\bar{u}_{c g_{i}}}{2 L_{g_{i}}} \bar{m}_{g_{i} q} \\
& 0=-\frac{R_{w_{j}}}{L_{w_{j}}} \bar{i}_{w_{j} d}+\omega_{w_{j}} \bar{i}_{w_{j} q}+\frac{\bar{v}_{s w_{j} d}}{L_{w_{j}}}-\frac{\bar{u}_{j}}{2 L_{w_{j}}} \bar{m}_{w_{j} d} \\
& 0=-\frac{R_{w_{j}}}{L_{w_{j}}} \bar{i}_{w_{j} q}-\omega_{w_{j}} \bar{i}_{w_{j} d}+\frac{\bar{v}_{s w_{j} q}}{L_{w_{j}}}-\frac{\bar{u}_{c w_{j}}}{2 L_{w_{j}}} \bar{m}_{w_{j} q} \\
& 0=\omega_{w_{j}} \bar{v}_{s w_{j} q}+\frac{1}{C_{f w_{j}}}\left(I_{w_{j} d}-\bar{i}_{w_{j} d}-\frac{\bar{v}_{s w_{j} d}}{R_{f w_{j}}}\right) \\
& 0=-\omega_{w_{j}} \bar{v}_{s w_{j} d}+\frac{1}{C_{f w_{j}}}\left(I_{w_{j} q}-\bar{i}_{w_{j} q}-\frac{\bar{v}_{s w_{j} q}}{R_{f w_{j}}}\right) \\
& 0=A_{p} \bar{z}+\bar{\vartheta}_{p}
\end{aligned}
$$

where $\bar{u}_{c g_{1}}=u_{c g_{1}}^{o}, \bar{i}_{g_{1} q}=-\frac{2 Q_{g_{1}}^{o}}{3 v_{s g_{1} d}}=0, \bar{i}_{g_{\rho} d}=\frac{2 P_{g_{\rho}}^{o}}{3 v_{s g_{1} d}}$, $\bar{i}_{g_{\rho} q}=-\frac{2 Q_{g_{\rho}}^{o}}{3 v_{s g_{1} d}}=0$ and $\bar{v}_{s w_{j}, d q}=v_{s w_{j}, d q}^{o}$.

Remark 2: Note that the above algebraic equations (13) contains several quadratic terms. It implies that the MTDC system has more than one equilibrium point that corresponds to the prescribed references [10], [20]. But, not all of these equilibrium points are achievable. As presented in [20], there are two possible steady-state values for the $d$-axis current. However, the larger one is physically impossible, making the converter operate beyond its ability. The choice of the proper equilibrium point is determined by the system physical characteristics, the feasibility of the modulation technique, etc. But this issue is beyond the scope of this paper.

To ensure the operating feasibility of the MTDC system, we make the following assumption on the equilibrium point $\bar{P}_{1}$.

Assumption 1: Consider the MTDC system described by (1), (4), (5) and (9). For the prescribed references $u_{c g_{1}}^{o}, Q_{g_{1}}^{o}$, $P_{g_{\rho}}^{o}, Q_{g_{\rho}}^{o}, v_{s w_{j}, d q}^{o}$, only one equilibrium point $\bar{P}_{1}$ exits in the domains of interest.

\footnotetext{
${ }^{2}$ See the notations
}

\section{B. Output control}

As presented in [9], according to the power direction, the control signal must be switched between two input-out feedback control laws, which may lead to some undesired effects. This shortcoming has been overcome in [10], [12] where a globally asymptotically stable controller is developed based on a passive output. However, due to the existence of uncontrolled internal dynamics, the convergence speed is less than satisfactory as illustrated in [10]. In this paper, another control algorithm based on the passive output is proposed, which has a nested structure. Because of the length limitation, the proofs of some results to be stated in the following part will not be presented.

By modifying the passive output given in [10], [12], we can deduce the following new output

$$
\begin{aligned}
& y=\left[\begin{array}{ll}
y_{g} & y_{w}
\end{array}\right]^{T} \\
& y_{g}=\left[\begin{array}{llllllll}
y_{g_{1} d} & y_{g_{1} q} & \cdots & y_{g_{i} d} & y_{g_{i} q} & \cdots & y_{g_{N} d} & y_{g_{N} q}
\end{array}\right]^{T}
\end{aligned}
$$

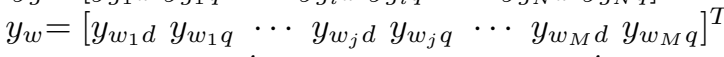

$$
\begin{aligned}
& y_{g_{i} d}=\alpha_{g_{i} d} u_{c g_{i}}-i_{g_{i} d} ; y_{g_{i} q}=\alpha_{g_{i} q} u_{c g_{i}}-i_{g_{i} q} \\
& y_{w_{j} d}=\alpha_{w_{j} d} u_{c w_{j}}-i_{w_{j} d} ; y_{w_{j} q}=\alpha_{w_{j} q} u_{c w_{j}}-i_{w_{j} q}
\end{aligned}
$$

where the variables of $\alpha_{g_{i}, d q}$ and $\alpha_{w_{j}, d q}$ are yet to be designed.

Lemma 1: Consider the system $\Sigma_{2}$ described by (1), (4), (5) and (9) with the output (14). The following algebraic equations

$$
\begin{aligned}
& 0=-\frac{R_{g_{i}}}{L_{g_{i}}} i_{g_{i} d}+\omega_{g_{i}} i_{g_{i} q}+\frac{v_{s g_{i} d}}{L_{g_{i}}}-\frac{u_{c g_{i}}}{2 L_{g_{i}}} m_{g_{i} d} \\
& 0=-\frac{R_{g_{i}}}{L_{g_{i}}} i_{g_{i} q}-\omega_{g_{i}} i_{g_{i} d}+\frac{v_{s g_{i} q}}{L_{g_{i}}}-\frac{u_{c g_{i}}}{2 L_{g_{i}}} m_{g_{i} q} \\
& 0=-\frac{R_{w_{j}}}{L_{w_{j}}} i_{w_{j} d}+\omega_{w_{j}} i_{w_{j} q}+\frac{v_{s w_{j}}}{L_{w_{j}}}-\frac{u_{c w_{j}}}{2 L_{w_{j}}} m_{w_{j} d} \\
& 0=-\frac{R_{w_{j}}}{L_{w_{j}}} i_{w_{j} q}-\omega_{w_{j}} i_{w_{j} d}+\frac{v_{s w_{j} q}}{L_{w_{j}}}-\frac{u_{c w_{j}}}{2 L_{w_{j}}} m_{w_{j} q} \\
& 0=\omega_{w_{j}} v_{s w_{j} q}+\frac{1}{C_{f w_{j}}}\left(I_{w_{j} d}-i_{w_{j} d}-\frac{v_{s w_{j} d}}{R_{f w_{j}}}\right) \\
& 0=-\omega_{w_{j}} v_{s w_{j} d}+\frac{1}{C_{f w_{j}}}\left(I_{w_{j} q}-i_{w_{j} q}-\frac{v_{s w_{j} q}}{R_{f w_{j}}}\right) \\
& 0=A_{p} z+\vartheta_{p} \\
& 0=y_{g_{i}, d q}=\alpha_{g_{i}, d q} u_{c g_{i}}-i_{g_{i}, d q} \\
& 0=y_{w_{j}, d q}=\alpha_{w_{j}, d q} u_{c w_{j}}-i_{w_{j}, d q}
\end{aligned}
$$

have at least one solution which is denoted by $(\cdot)^{\alpha}$ and the set of the equilibrium points is denoted by $\mathcal{P}_{\alpha}$. In particular, if $\alpha_{g_{i}, d q}$ and $\alpha_{w_{j}, d q}$ are given by

$$
\begin{aligned}
\alpha_{g_{i}, d q} & =\frac{\bar{i}_{g_{i}, d q}}{\bar{u}_{c g_{i}}} \\
\alpha_{w_{j}, d q} & =\frac{\bar{i}_{w_{j}, d q}}{\bar{u}_{c w_{j}}}
\end{aligned}
$$

there exists $\bar{P}_{2} \in \mathcal{P}_{\alpha}$ such that $\bar{P}_{2}=\bar{P}_{1}$.

Remark 3: The above lemme shows that, if $\alpha_{g_{i}, d q}$ and $\alpha_{w_{j}, d q}$ are properly chosen, the steady-state values of $\Sigma_{2}$ could consist with those prescribed values.

For the system $\Sigma_{2}$, we have the following result.

Proposition 1: Consider the system $\Sigma_{2}$ and assume that there exists an equilibrium $\bar{P}_{2}$ whose elements $u_{c g_{i}}^{\alpha}, u_{c w_{j}}^{\alpha}$ and 
$u_{c t_{h}}^{\alpha}$ are positive. Then, a new system $\tilde{\Sigma}_{2}$ can be obtained by shifting $\bar{P}_{2}$ to the origin. The new system $\tilde{\Sigma}_{2}$ is

- passive with a radially unbounded positive definite storage function and

- zero-state observable in the set $\{y=0\}$.

The control law

$$
\begin{aligned}
m_{g_{i} d} & =-c_{g_{i} d, 0} \cdot y_{g_{i} d}-k_{I g_{i} d} \cdot e_{I y_{g_{i} d}} \\
m_{g_{i} q} & =-c_{g_{i} q, 0} \cdot y_{g_{i} q}-k_{I g_{i} q} \cdot e_{I y_{g_{i} q}} \\
m_{w_{j} d} & =-c_{w_{j} d, 0} \cdot y_{w_{j} d}-k_{I w_{j} d} \cdot e_{I y_{w_{j} d}} \\
m_{w_{j} q} & =-c_{w_{j} q, 0} \cdot y_{w_{j} q}-k_{I w_{j} q} \cdot e_{I y_{w_{j} q}}
\end{aligned}
$$

with positive control gains $c_{(\cdot)}$ and $k_{I(\cdot)}$ and the integrated tracking errors

$$
\begin{gathered}
\dot{e}_{I y_{g_{i} d, q}}=y_{g_{i} d, q} ; \\
\dot{e}_{I y_{w_{j} d, q}}=y_{w_{j} d, q}
\end{gathered}
$$

can asymptotically stabilize the origin of $\tilde{\Sigma}_{2}$ and the equilibrium $\bar{P}_{2}$ of $\Sigma_{2}$.

\section{Outer control loop: control of zero dynamics}

Substituting the output control algorithm (17) into the system $\Sigma_{2}$, the dynamics of the DC grid and the AC voltages $v_{s w_{j}, d q}$ will be governed by the corresponding zero dynamics when the output variables $y_{g_{i}, d q}$ and $y_{w_{i}, d q}$ perfectly converge to zero. Therefore, by controlling $y$ at zero, we can indirectly drive $u_{c g_{1}}, i_{g_{\rho} d}, i_{g_{i} q}$ and the AC voltage $v_{s w_{j}, d q}$ to the corresponding steady states $(\cdot)^{\alpha}$. As stated in Remark 3, in order to make the steady-state values of $\Sigma_{2}$ consist with those prescribed reference values, $\alpha_{(\cdot)}$ should be given the right values (see (16)) which are calculated from (13). According to (3) and (12), we can easily obtain $\bar{i}_{g_{i} q}=0$ and hence $\alpha_{g_{i} q}=\frac{\bar{i}_{g_{i} q}}{\bar{u}_{c g_{i}}}=0$ can be directly obtained. However, there is still a need to deduce $\alpha_{g_{i} d}$ and $\alpha_{w_{j}, d q}$ by solving (13). Due to the uncertainties on the system parameters or unmodeled elements in the power system, it is usually difficult to get the accurate values of $\alpha_{g_{i} d}=\frac{\bar{i}_{g_{i} d}}{\bar{u}_{c g_{i}}}$ and $\alpha_{w_{j}, d q}=\frac{\bar{i}_{w_{j}, d q}}{\bar{u}_{c w_{j}}}$ and then steady-state errors may exist. Furthermore, the convergence rate of the uncontrolled zero dynamics totally depends on the system inherent characteristics, which could be very slow. Therefore, we expect to regulate the behaviors of the zero dynamics and to achieve good tracking performance, i.e. to make $u_{c g_{1}}, i_{g_{\rho} d}, i_{g_{i} q}$ and the AC voltage $v_{s w_{j}, d q}$ follow their respective prescribed values $(\cdot)^{o}$ with a desired convergence rate. This will be achieved by designing $\alpha_{g_{i} d}$ and $\alpha_{w_{j}, d q}$ from an outer control loop which is based on the zero dynamics of the system $\Sigma_{2}$.

Remark 4: In the following part, we consider the case where $\bar{i}_{g_{\rho} d} \neq 0$. If $P_{g_{\rho} d}^{o}$ are set to zero for some SAC terminals, then

$$
\alpha_{g_{\rho} d}=\frac{\bar{i}_{g_{i} d}}{\bar{u}_{c g_{i}}}=\frac{2 P_{g_{\rho}}^{o}}{3 \bar{u}_{c g_{\rho}} v_{s g_{\rho} d}}=0
$$

can be directly deduced without further design.
As the output vector $y$ approaches zero, the zero dynamics of the system $\Sigma_{2}$ can be deduced as

$$
\begin{aligned}
& \frac{d v_{s w_{j} d}}{d t}=\omega_{w_{j}} v_{s w_{j} q}+\frac{1}{C_{f w_{j}}}\left(I_{w_{j} d}-\alpha_{w_{j} d}^{*} u_{c w_{j}}-\frac{v_{s w_{j} d}}{R_{f w_{j}}}\right) \\
& \frac{d v_{s w_{j} q}}{d t}=-\omega_{w_{j}} v_{s w_{j} d}+\frac{1}{C_{f w_{j}}}\left(I_{w_{j} q}-\alpha_{w_{j} q}^{*} u_{c w_{j}}-\frac{v_{s w_{j} q}}{R_{f w_{j}}}\right) \\
& C_{g_{1}} \frac{d u_{c g_{1}}}{d t}=\frac{\frac{1}{2 L_{g_{1}}}}{\frac{1}{2 L_{g_{1}}}+\frac{3}{4}\left(\alpha_{g_{1} d}^{*}\right)^{2}}\left\{\left[-\frac{u_{c g_{1}}}{R_{\text {equi } g_{1}}}+H(1,:) i_{c}\right]\right. \\
& \left.-\frac{3}{2}\left[R_{g_{1}} u_{c g_{1}}\left(\alpha_{g_{1} d}^{*}\right)^{2}-v_{s g_{1} d} \alpha_{g_{1} d}^{*}\right]\right\} \\
& C_{g_{\rho}} \frac{d i_{g_{\rho} d}}{d t}=\frac{\frac{1}{2 L_{g_{\rho}}}}{\frac{1}{2 L_{g_{\rho}}}+\frac{3}{4}\left(\alpha_{g_{\rho} d}^{*}\right)^{2}}\left\{\left[-\frac{i_{g_{\rho} d}}{R_{\text {equi }, g_{\rho}}}+\alpha_{g_{\rho} d}^{*} H(i,:) i_{c}\right]\right. \\
& \left.-\frac{3}{2}\left[R_{g_{\rho}} i_{g_{\rho} d}\left(\alpha_{g_{\rho} d}^{*}\right)^{2}-\alpha_{g_{\rho} d}^{*}\left(v_{s g_{\rho} d} \alpha_{g_{\rho} d}^{*}\right)\right]\right\} \\
& C_{w_{j}} \frac{d u_{c w_{j}}}{d t}=\frac{\frac{1}{2 L_{w_{j}}}}{\frac{1}{2 L_{w_{j}}}+\frac{3}{4}\left[\left(\alpha_{w_{j} d}^{*}\right)^{2}+\left(\alpha_{w_{j} q}^{*}\right)^{2}\right]}\left\{\left[-\frac{u_{c w_{j}}}{R_{\text {equi } w_{j}}}\right.\right. \\
& \left.+H(N+j,:) i_{c}\right]-\frac{3}{2}\left[R _ { w _ { j } } u _ { c w _ { j } } \left(\left(\alpha_{w_{j} d}^{*}\right)^{2}\right.\right. \\
& \left.\left.\left.+\left(\alpha_{w_{j} q}^{*}\right)^{2}\right)-\left(v_{s w_{j} d} \alpha_{w_{j} d}^{*}+v_{s w_{j} q} \alpha_{w_{j} q}^{*}\right)\right]\right\} \\
& C_{t_{h}} \frac{d u_{c t_{h}}}{d t}=H(N+M+h,:) i_{c} \\
& L_{c_{k}} \frac{d i_{c k}}{d t}=-R_{c_{k}} i_{c_{K}}-H(:, k)^{T} u_{c}
\end{aligned}
$$

Now, the zero dynamics (19) is considered as a new system where $\alpha_{g_{i} d}$ and $\alpha_{w_{j}, d q}$ are replaced by $\alpha_{g_{i} d}^{*}$ and $\alpha_{w_{j}, d q}^{*}$ to emphasize that they are considered as control inputs. It can be seen that the number of control variables $\left(\alpha_{g_{i} d}^{*}\right.$ and $\left.\alpha_{w_{j}, d q}^{*}\right)$ equals the number of controlled variables $\left(u_{c g_{1}}, i_{g_{\rho} d}\right.$ and $\left.v_{s w_{j}, d q}\right)$ and moreover, $\alpha_{w_{j}, d q}^{*}$ are directly collocated with $v_{s w_{j}, d q}$. We can use two steps to design these auxiliary control variables.

1) Design of $\alpha_{w_{j}, d q}^{*}$ : We first design $\alpha_{w_{j}, d q}^{*}$ to make $v_{s w_{j}, d q}$ in (19) track their reference values $v_{s w_{J}, d q}^{o}$ while $\alpha_{g_{i} d}^{*}$ are considered constant. It is natural to choose the output as

$$
\begin{aligned}
y_{w} & =\left[\begin{array}{lll}
y_{w_{1}} & \cdots & y_{w_{M}}
\end{array}\right]^{T} \\
y_{w_{j}} & =\left[\begin{array}{lll}
v_{s w_{j} d} & v_{s w_{j} q}
\end{array}\right]^{T}
\end{aligned}
$$

We then have the following result.

Lemma 2: Consider the system $\Sigma_{3}$ described by (19) with the output $y_{w}$ defined by (20) where $\alpha_{g_{i} d}^{*}$ are constant and $\alpha_{w_{j}, d q}^{*}$ are the control inputs. By restricting

$$
v_{s w_{j}, d q} \equiv v_{s w_{j}, d q}^{o}
$$


the zero dynamics of $\Sigma_{3}$ can be characterized by

$$
\begin{aligned}
& C_{g_{1}} \frac{d u_{c g_{1}}}{d t}=\frac{\frac{1}{2 L_{g_{1}}}}{\frac{1}{2 L_{g_{1}}}+\frac{3}{4}\left(\alpha_{g_{1} d}^{*}\right)^{2}}\left\{\left[-\frac{u_{c g_{1}}}{R_{\text {equi, } g_{1}}}+H(1,:) i_{c}\right]\right. \\
& \left.-\frac{3}{2}\left[R_{g_{1}} u_{c g_{1}}\left(\alpha_{g_{1} d}^{*}\right)^{2}-v_{s g_{1} d} \alpha_{g_{1} d}^{*}\right]\right\} \\
& C_{g_{\rho}} \frac{d i_{g_{\rho} d}}{d t}=\frac{\frac{1}{2 L_{g_{\rho}}}}{\frac{1}{2 L_{g_{\rho}}}+\frac{3}{4}\left(\alpha_{g_{\rho} d}^{*}\right)^{2}}\left\{\left[\alpha_{g_{\rho} d}^{*} H(\rho,:) i_{c}-\frac{i_{g_{\rho} d}}{R_{\text {equi }, g_{\rho}}}\right]\right. \\
& \left.-\frac{3}{2}\left[R_{g_{\rho}} i_{g_{\rho} d}\left(\alpha_{g_{\rho} d}^{*}\right)^{2}-\alpha_{g_{\rho} d}^{*}\left(v_{s g_{\rho} d} \alpha_{g_{\rho} d}^{*}\right)\right]\right\} \\
& C_{w_{j}} \frac{d u_{c w_{j}}}{d t}=\frac{\frac{1}{2 L_{w_{j}}}}{\frac{1}{2 L_{w_{j}}}+\frac{3}{4}\left[\left(\bar{\alpha}_{w_{j} d}^{*}\right)^{2}+\left(\bar{\alpha}_{w_{j} q}^{*}\right)^{2}\right]}\left\{\left[-\frac{u_{c w_{j}}}{R_{\text {equi }, w_{j}}}\right.\right. \\
& \left.+H(N+j,:) i_{c}\right]-\frac{3}{2}\left[R_{w_{j}} u_{c w_{j}}\left(\left(\bar{\alpha}_{w_{j} d}^{*}\right)^{2}+\left(\bar{\alpha}_{w_{j} q}^{*}\right)^{2}\right)\right. \\
& \left.\left.-\left(v_{s w_{j} d}^{o} \bar{\alpha}_{w_{j} d}^{*}+v_{s w_{j} q}^{o} \bar{\alpha}_{w_{j} q}^{*}\right)\right]\right\} \\
& C_{t_{h}} \frac{d u_{c t_{h}}}{d t}=H(N+M+h,:) i_{c} \\
& L_{c_{k}} \frac{d i_{c k}}{d t}=-R_{c_{k}} i_{c_{K}}-H(:, k)^{T} u_{c}
\end{aligned}
$$

where $\bar{\alpha}_{w_{j}, d q}^{*}$ satisfy

$$
\bar{\alpha}_{w_{j} d, q}^{*}=\frac{1}{u_{c w_{j}}} \bar{i}_{w_{j} d, q}
$$

The equilibrium of (21) is locally asymptotically stable. Thus, $\Sigma_{3}$ is minimum phase.

As a result, by following the input-output feedback linearization procedure, $\alpha_{w_{j}, d q}$ can be developed as

$$
\begin{aligned}
& \alpha_{w_{j} d}^{*}=\frac{1}{u_{c w_{j}}}\left[I_{w_{j} d}-\frac{v_{s w_{j} d}}{R_{f w_{j}}}+C_{f w_{j}} \omega_{w_{j}} v_{s w_{j} q}-C_{f w_{j}} v_{\alpha_{w_{j}}}\right] \\
& \alpha_{w_{j} q}^{*}=\frac{1}{u_{c w_{j}}}\left[I_{w_{j} q}-\frac{v_{s w_{j} q}}{R_{f w_{j}}}-C_{f w_{j}} \omega_{w_{j}} v_{s w_{j} d}-C_{f w_{j}} v_{\alpha_{w_{j} q}}\right]
\end{aligned}
$$

where

$$
\begin{aligned}
\dot{e}_{I \alpha_{w_{j}}} & =e_{v_{s w_{j} d}} \triangleq v_{s w_{j} d}-v_{s w_{j} d}^{o} \\
v_{\alpha_{w_{j}} d} & =-c_{\alpha_{w_{j} d}} \cdot e_{v_{s w_{j} d}}-k_{I \alpha_{w_{j} d}} \cdot e_{I \alpha_{w_{j} d}} \\
\dot{e}_{I \alpha_{w_{j} q}} & =e_{v_{s w_{j} q}} \triangleq v_{s w_{j} q}-v_{s w_{j} q}^{o} \\
v_{\alpha_{w_{j} q}} & =-c_{\alpha_{w_{j} q}} \cdot e_{v_{s w_{j} q}}-k_{I \alpha_{w_{j} q}} \cdot e_{I \alpha_{w_{j} q}}
\end{aligned}
$$

with positive control gains $c_{\alpha_{w_{j}, d q}}$ and $k_{I \alpha_{w_{j}, d q}}$.

2) Design of $\alpha_{g_{i} d}^{*}$ : From the previous section, we get that, when $v_{s w_{j}, d q}$ converge to $v_{s w_{j} d q}^{o}$, the behavior of the system $\Sigma_{3}$ is governed by the corresponding zero dynamics (21) whose state variables, $u_{c g_{1}}, i_{g_{\rho}}, u_{c w_{j}}, u_{c t_{h}}$ and $i_{c}$, are uncontrolled. Again, we want to regulate these desired controlled variables, i.e. $u_{c g_{1}}$ and $i_{g_{\rho}}$, by designing $\alpha_{g_{i} d}^{*}$.

In this part, the algorithm for $\alpha_{g_{i} d}^{*}$ is derived from the zero dynamics (21) where $\alpha_{g_{i} d}^{*}$ are considered as control variables. To get good tracking performance, the PI control method is applied to design $\alpha_{g_{i} d}^{*}$.

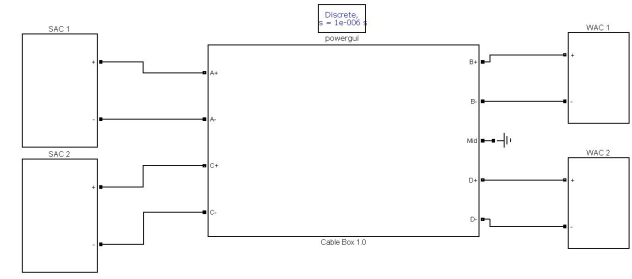

Fig. 5. An MTDC system consists of two strong and two weak AC systems.

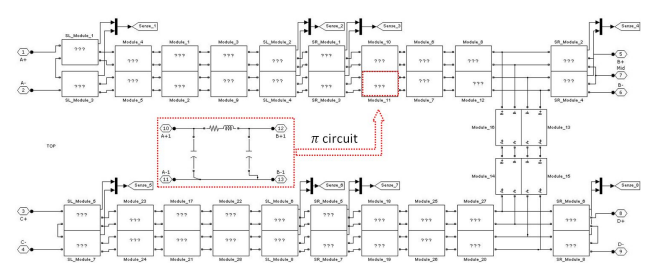

Fig. 6. DC grid used to connect the four AC areas.

Theorem 1: Consider the system $\Sigma_{4}$ described by (21) with the control variables $\alpha_{g_{i} d}^{*}$. There exist some standard PI controllers such that

$$
\begin{aligned}
\dot{e}_{I u_{c g_{1}}} & =\tilde{u}_{c g_{1}} \\
\alpha_{g_{1} d}^{*} & =-k_{P u_{c g_{1}}} \tilde{u}_{c g_{1}}-k_{I u_{c g_{1}}} e_{I u_{c g_{1}}} \\
\dot{e}_{I i_{g_{\rho} d}} & =\tilde{i}_{g_{\rho} d} \\
\alpha_{g_{\rho} d}^{*} & =-k_{P i_{g_{\rho} d}} \tilde{i}_{g_{\rho} d}-k_{I i_{g_{\rho} d}} e_{I i_{g_{\rho} d}}
\end{aligned}
$$

with the integrated errors and positive control gains $k_{I(\cdot)}$, can locally stabilize the equilibrium of the system $\Sigma_{4}$.

Finally, we can state the following result.

Theorem 2: Consider the MTDC system modeled by (1), (4), (5) and (9). The proposed control algorithm which is composed of (17), (23) and (24) can locally stabilize the equilibrium point $\bar{P}_{1}$.

\section{Simulation studies}

To evaluate the proposed nonlinear controller, numerical simulations are carried out by using SimPowerSystems toolbox of MATLAB/Simulink as depicted in Fig. 5 where an MTDC system consists of two SAC, two WAC connected VSC terminals and a DC network (Fig. 6). In addition, the feedback nonlinear controller proposed in [9] is also tested for comparison.

\section{A. Scenario 1: DC voltage regulation and power reversal operation}

Initially, the MTDC system works in the steady state as illustrated in Table I. At $t=0.5 \mathrm{~s}, i_{g_{2} d}^{o}$ is set to $-6.53 \mathrm{~A}$ from $13.1 \mathrm{~A}$. It means that the $2^{\text {nd }} \mathrm{SAC}$ connected VSC is required to operate in the inversion mode as a power consumer from the rectification mode as a power supplier. Then, at $t=1 \mathrm{~s}$, a new reference value of $u_{c g_{1}}$ is given with an increase of $5 \%$.

The simulation results are shown in Fig. 7. As plotted in Fig. 7(a), both controllers can make $u_{c g_{1}}$ always track its reference $u_{c g_{1}}^{o}$. However, when the power reversal happens in 
TABLE I

INITIAL VALUES OF THE SYSTEM STATE VARIABLES.

\begin{tabular}{|c|c|}
\hline SAC 1 & SAC 2 \\
\hline$u_{c g_{1}}=u_{c g_{1}}^{o}=150 \mathrm{~V}$ & $i_{g_{2} d}=i_{g_{2} d}^{o}=13.1 \mathrm{~A}$ \\
\hline$i_{g_{1} q}=i_{g_{1} q}^{o}=0 \mathrm{~A}$ & $i_{g_{2} q}=i_{g_{2} q}^{o}=0 \mathrm{~A}$ \\
\hline \hline WAC 1 & WAC 2 \\
\hline$v_{s w_{1} d}=v_{s w_{1} d}^{o}=40.82 \mathrm{~V}$ & $v_{s w_{2} d}=v_{s w_{2} d}^{o}=40.82 \mathrm{~V}$ \\
\hline$v_{s w_{1} q}=v_{s w_{1} q}^{o}=0 \mathrm{~V}$ & $v_{s w_{2} q}=v_{s w_{2} q}^{o}=0 \mathrm{~V}$ \\
\hline
\end{tabular}

the $2^{\text {nd }}$ SAC connected VSC terminal, the trajectory of $u_{c g_{1}}$ under the feedback nonlinear controller drops very fast and has an unacceptable undershoot. Compared to the feedback nonlinear controller, the proposed controller gives a much better performance with a faster convergence and keeps $u_{c g_{1}}$ within its acceptable region. As illustrated in Figs. 7(b) and 7(c), the changes of $u_{c g_{1}}^{o}$ and $i_{g_{2} d}^{o}$ have negligible effects on the performance of $i_{g_{1} q}$ (and $i_{g_{2} q}$ ) under feedback nonlinear controller while remarkable overshoot (and undershoot) appears when the proposed controller is used. These phenomena imply that the feedback nonlinear controller always provides a better decoupling characteristics of the DC voltage (or the $\mathrm{AC} d$ - axis current) and the $\mathrm{AC} q-$ axis current control than the proposed controller.

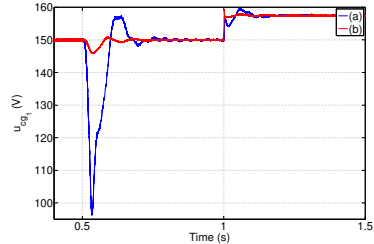

(a) Response of $u_{c g_{1}}$.

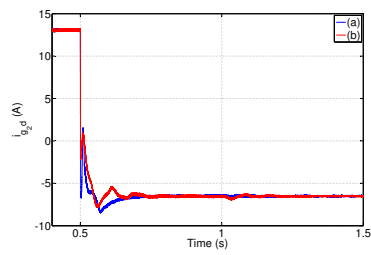

(c) Response of $i_{g_{2} d}$.

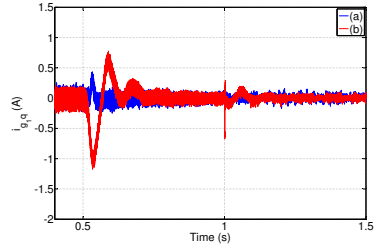

(b) Response of $i_{g_{1} q}$.

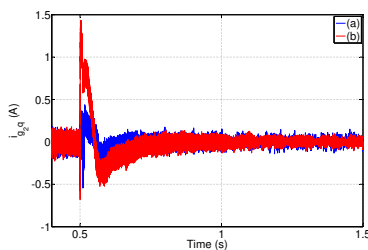

(d) Response of $i_{g_{2} q}$.
Fig. 7. Scenario 1: Simulation results (1) Feedback nonlinear controller (red curve). (2) Proposed controller (blue curve).

\section{B. Scenario 2: AC voltage regulation}

To evaluate the capability of the proposed controller in terms of AC voltage regulation, the step changes of $v_{s w_{1} d}^{o}$ and $v_{s w_{2} q}^{o}$ are considered in this scenario. Similar to Scenario 1 , the MTDC system initially operates in the steady state provided by Table I. At $t=0.5 \mathrm{~s}$ and $t=1 \mathrm{~s}, v_{s w_{1} d}^{o}$ and $v_{s w_{2} q}^{o}$ are changed to $61.24 \mathrm{~V}$ and $20.41 \mathrm{~V}$ respectively. The simulation results are plotted in Figs. 8. The transient response of $u_{c g_{1}}$ is illustrated in Fig. 8(a). It is evident that the performance of $u_{c g_{1}}$ under the proposed controller is much better than the feedback nonlinear controller. Less oscillations, smaller overshoots and faster response convergence are found in the trajectory of $u_{c g_{1}}$ under the proposed controller. Similar to the results in Scenario 1, Figs. 8(b)-8(e) clearly show that the feedback nonlinear controller gives a better decoupling characteristics of the $\mathrm{AC} d-$ axis and $q-$ axis voltage control.

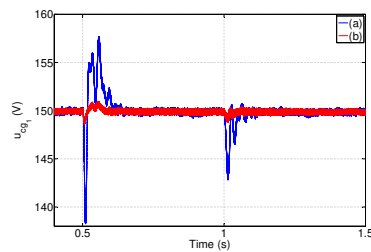

(a) Response of $u_{c g_{1}}$.

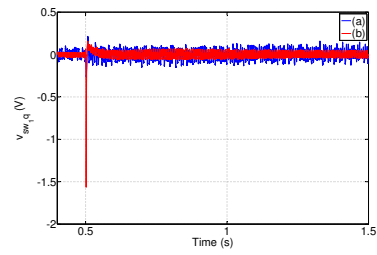

(c) Response of $v_{s w_{1} q}$.

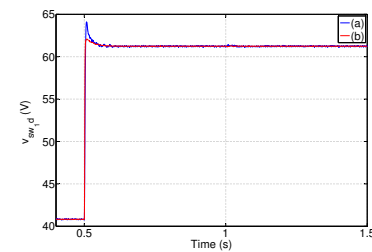

(b) Response of $v_{s w_{1} d}$

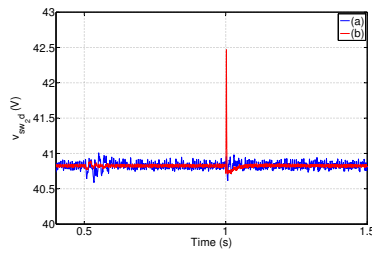

(d) Response of $v_{s w_{2} d}$.

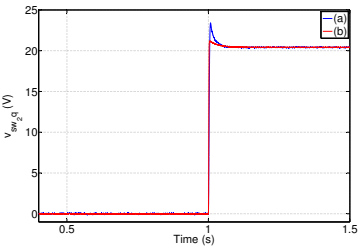

(e) Response of $v_{s w_{2} q}$

Fig. 8. Scenario 2: Simulation results (1) Feedback nonlinear controller (red curve). (2) Proposed controller (blue curve).

In addition, from the above numerical simulation results, the convergence rate of the system state variables are clearly improved compared to simulation results in [10] where just the passive control law is used without the extra outer control loop.

\section{Conclusions}

This paper proposes a nonlinear controller based on a passive output and an outer control loop. We point out that if only the output controller is applied to the MTDC system, the dynamics of the internal states, which are also called the zero dynamics, are uncontrolled. However, the zero dynamics contain some desired control variables such as the $\mathrm{AC}$ voltages of the wind farms at the PCCs, the DC voltage etc. In order to improve the performances of those desired control variables, the outer control loop is designed to control the zero dynamics, which provides the way to regulate the output controller's parameters. Simulation results show that the convergence speed of the system states is greatly improved. Moreover, in comparison, the nonlinear feedback linearization controller performs worse than the proposed nonlinear controller in case of power reversal.

\section{REFERENCES}

[1] W. Long and S. Nilsson, "HVDC transmission: yesterday and today," IEEE Power and Energy Magazine, vol. 5, pp. 22-31, Mar 2007. 
[2] B. T. Ooi and X. Wang, "Voltage angle lock loop control of the boost type pwm converter for hvdc application," IEEE Transactions on Power Electronics, vol. 5, pp. 229-235, Apr 1990.

[3] T. Noguchi, H. Tomiki, S. Kondo, and I. Takahashi, "Direct power control of pwm converter without power-source voltage sensors," IEEE Transactions on Industry Applications, vol. 34, May 1998.

[4] A. Lindberg and L. Lindberg, "Inner current loop for large voltage low switching frequency," in Fifth International Conference on Power Electronics and Variable-Speed Drives, pp. 217-222, Oct 1994.

[5] V. Blasko and V. Kaura, "A new mathematical model and control of a three-phase AC-DC voltage source converter," IEEE Transactions on Power Electronics, vol. 12, pp. 116-123, Jan 1997.

[6] C. Bajracharya, M. Molinas, J. A. Suul, T. M. Undeland, et al., "Understanding of tuning techniques of converter controllers for VSCHVDC," in Nordic Workshop on Power and Industrial Electronics, (Espoo, Finland), Helsinki University of Technology, Jun 2008.

[7] G. Beccuti, G. Papafotiou, and L. Harnefors, "Multivariable predictive control of voltage source converter HVDC transmission systems," in IEEE International Symposium on Industrial Electronics (ISIE),, pp. 3145-3152, Jul 2010.

[8] D.-E. Kim and D.-C. Lee, "Feedback linearization control of three-phase UPS inverter systems," IEEE Transactions on Industrial Electronics, vol. 57, pp. 963-968, Mar 2010.

[9] Y. Chen, G. Damm, A. Benchaib, and F. Lamnabhi-Lagarrigue, "Feedback linearization for the DC voltage control of a VSC-HVDC terminal," in 2014 European Control Conference (ECC), pp. 1999-2004, Jun 2014.

[10] D. Zonetti, R. Ortega, and A. Benchaib, "Modeling and control of highvoltage direct-current transmission systems: From theory to practice and back," Automatica, 2014.

[11] I. Martinez-Perez, G. Espinosa-Perez, G. Sandoval-Rodriguez, and A. Doria-Cerezo, "IDA passivity-based control of single phase backto-back converters," in IEEE International Symposium on Industrial Electronics, pp. 74-79, 2008.

[12] M. Hernandez-Gomez, R. Ortega, F. Lamnabhi-Lagarrigue, and G. Escobar, "Adaptive PI stabilization of switched power converters," IEEE Transactions on Control Systems Technology, vol. 18, pp. 688-698, May 2010.

[13] G. Escobar, A. J. Van Der Schaft, and R. Ortega, "A hamiltonian viewpoint in the modeling of switching power converters," Automatica, vol. 35, no. 3, pp. 445-452, 1999.

[14] L. Xu, L. Yao, and C. Sasse, "Grid integration of large dfig-based wind farms using VSC transmission," IEEE Transactions on Power Systems, vol. 22, pp. 976-984, Aug 2007.

[15] R. Pena, J. Clare, and G. Asher, "Doubly fed induction generator using back-to-back PWM converters and its application to variable-speed wind-energy generation," IEE Proceedings - Electric Power Application$s$, vol. 143, pp. 231-241, May 1996.

[16] X. Lie, B. Williams, and Y. Liangzhong, "Multi-terminal DC transmission systems for connecting large offshore wind farms," in IEEE Power and Energy Society General Meeting - Conversion and Delivery of Electrical Energy in the 21st Century, pp. 1-7, Jul 2008.

[17] E. Prieto-Araujo, F. Bianchi, A. Junyent-Ferre, and O. Gomis-Bellmunt, "Methodology for droop control dynamic analysis of multiterminal VSC-HVDC grids for offshore wind farms," IEEE Transactions on Power Delivery, vol. 26, pp. 2476-2485, Oct 2011.

[18] J. Beerten, S. Cole, and R. Belmans, "Modeling of multi-terminal VSC HVDC systems with distributed DC voltage control," IEEE Transactions on Power Systems, vol. 29, pp. 34-42, Jan 2014.

[19] N. Mohan and T. M. Undeland, Power Electronics: Converters, Applications, and Design. John Wiley \& Sons Inc, 2003.

[20] T.-S. Lee, "Input-output linearization and zero-dynamics control of three-phase ac/dc voltage-source converters," IEEE Transactions on Power Electronics, vol. 18, pp. 11-22, Jan 2003. 